Propuesta didÁctica PARA LA ENSEÑANZA dE LOS MÉTOdOS PARA RESOLVER UN SISTEMA DE ECUACIONES LINEALES

\title{
Propuesta didáctica para la enseñanza de los métodos para resolver un sistema de ecuaciones lineales ${ }^{1}$
}

\author{
Didactic proposal for teaching the methods for
} solving a system of linear equations

\author{
Proposta didática para o ensino de métodos para \\ resolver um sistema de equações lineares
}

Recibido: mayo de 2013

Aceptado: agosto de 2013
Jorge Alejandro Rojas Gómez ${ }^{2}$ Aura Alejandra Ariza Daza ${ }^{3}$

\section{Resumen}

Este artículo pretende evidenciar la importancia que tiene la enseñanza de los métodos para resolver sistemas de ecuaciones lineales, de manera posterior a la función lineal, para esto se trabajará como metodología la teoría de situaciones didácticas de Brousseau, buscando la construcción propia de cada estudiantes, generando en ellos un aprendizaje significativo; esto se buscará a partir de un contexto familiar para ellos, impulsando las buenas aptitudes de los estudiantes y el ánimo para envolverse dentro de la situación.

Palabras clave: Matemáticas escolares; álgebra; ecuaciones; sistema de ecuaciones; Teoría de situaciones didácticas; enseñanza; planificación del profesor; metodología de enseñanza; metodología de trabajo en el aula.

\begin{abstract}
This article aims to show the importance of teaching the methods for solving systems of linear equations, so back to the linear function to this methodology will work as the theory of didactic situations of Brousseau, seeking to build its own of each student, generating meaningful learning them, that will search from a context familiar to them, encouraging the good skills of students and encouragement to get involved in the situation.
\end{abstract}

Keywords: school Mathematics, algebra equations, system of equations, Theory of didactic situations, teaching, teacher planning, teaching methodology, methodology of work in the classroom.

\section{Resumo}

Este artigo tem como objetivo mostrar a importância de ensinar os métodos de sistemas de equações lineares, então de volta para a função linear para resolver esta metodologia vai funcionar como a teoria das situações didáticas de Brousseau, buscando construir o seu próprio de cada aluno, gerando aprendizagem significativa deles, que irá procurar a partir de um contexto

1 Artículo de Investigación.

2 Universidad Distrital Francisco José de Caldas. Bogotá, Colombia. Contacto: lalorojas10@hotmail.es

3 Universidad Distrital Francisco José de Caldas. Bogotá, Colombia. Contacto: aleja9312@gmail.com 
familiar para eles, incentivando as boas habilidades dos alunos e incentivo para se envolver na situação.

Palavras-chave: Matemática da escola de álgebra, equações, sistema de equações, teoria das situações didáticas, pedagógicas, planejamento de professores, metodologia de ensino, metodologia de trabalho em sala de aula.

\section{Contextualización}

La presente experiencia de aula se llevará a cabo en el Colegio José Félix Restrepo sede A, y específicamente en el grado noveno, tendiendo como labor el impulsar en los estudiantes la manera de entender y resolver un sistema de ecuaciones lineales, para esto se decidió trabajar la TSD debido a que permiten la construcción de un conocimiento por los estudiantes a partir de un medio o contexto, otro aspecto importante que se resalta de estas, es la amplia cantidad de momentos de construir el conocimiento, teniendo en cuenta que llamamos momentos a cada una de las fases que esta teoría propone.

\section{Marco teórico}

Una función es lineal si y solo si puede escribirse de la forma en donde a, b son constantes, y . (Haussler, 2003, p.139). Igualmente una función lineal se puede considerar como: "Una función de proporcionalidad directa los valores que toman las variables y son en general números reales, que corresponden a las medidas de magnitudes que intervienen en las diversas situaciones" (Godino \& Font, 2003, pág. 803).

Esta tiene como dominio y codominio todos lo reales (trabajando en ese conjunto), y como se mostró anteriormente su expresión analítica es una ecuación de primer grado o lineal la cual es aquella que responde a la forma:

Existen dos tipos de ecuaciones lineales:

- Si la ecuación se denomina homogénea

- Si la ecuación se denomina no homogénea o completa
Cuando existe un conjunto de ecuaciones lineales donde se quiere que y tomen el mismo valor se llama sistema de ecuaciones lineales. En este caso se especificara el estudio de sistemas de ecuaciones lineales es decir, de dos ecuaciones, con dos incógnitas.

Y se deben encontrar el valor de las incógnitas y tales que las dos ecuaciones sean verdaderas. (Kolman, 2006, p.62), generalmente en situaciones reales se hace necesario utilizar ecuaciones lineales de primer grado (dos variables) y en otras se requieren dos o más ecuaciones lineales a la vez lo que se denomina sistema de ecuaciones lineales. Las ecuaciones con una incógnita, llamadas de primer grado reciben este nombre porque el máximo exponente de la variable independiente es 1.

En un sistema de ecuación lineal se pueden evidenciar tres casos: el sistema tiene una única solución, el sistema no tiene solución y el sistema tiene más de una solución (infindad de soluciones), en este caso solo se trabajará el primero. Solucionar un sistema de ecuaciones lineales significa encontrar los valores solución, que son comunes a todas las ecuaciones del sistema. Existen muchos métodos para resolver un sistema de ecuaciones lineales, pero en este caso solo trabajaremos cuatro los cuales son método gráfico, método de igualación, método de sustitución y el método de eliminación o reducción, expuestos por Gallego \& Fornés (2009).

El grupo Azarquiel, (1993) se evidencia que los estudiantes tienen dificultades a la hora de resolver un sistema de ecuaciones lineales:

En el método de igualación, el estudiante deja de por medio la ecuación inicial y la igualdad existente entre las dos ecuaciones, lo cual se debe a un aprendizaje muy pobre y difícil de resolver. Los estudiantes preparan correctamente las ecuaciones para reducirlas, y encuentra correctamente un valor 
el cual luego lo deja de lado. Y termina contradiciéndose en sus resultados. Igualmente algunos de los estudiantes solo tienen en cuenta una ecuación y dejan de lado la otra. (Grupo Azarquiel, 1993.p. 111-112)

Una de las principales dificultades es que los estudiantes no identifican el método más fácil o pertinente para dar solución a un sistema de ecuaciones, por lo que el estudiante llega a eliminar una ecuación o dejar de lado una incógnita. Además los estudiantes presentan dificultad al separar las informaciones dependientes, o designar con distintas letras incógnitas diferentes (Grupo Azarquiel, 1993.p.115).Y otros errores son ya de cálculo, que los estudiantes dejan de lado, o por que un resultado llegue a ser contradictorio.

Pero estos errores tienen un trasfondo más profundo, el cual se puede deber a que no se tiene un concepto claro y una manipulación de las ecuaciones de primer grado, lo cual esta dado por la relación conceptual entre las nociones de incógnita y ecuación, y las de variable y función.

\section{Descripción general}

La experiencia de aula que se quiere compartir tiene lugar en la ciudad de Bogotá y específicamente en el grado noveno del colegio José Félix Restrepo (sede A). Inicialmente se hizo la aplicación de una prueba diagnóstico, la cual pretendía ver los conceptos previos a la enseñanza y el aprendizaje de la función lineal y los sistemas de ecuaciones los cuales eran: correlación \& variación directa e inversa, despeje a partir de inversos (en $Q_{-} Z$ ) y el uso de la letra como incógnita.

Los resultados obtenidos se evidenció que los estudiantes aun tienen complicaciones al realizar despejes, ya que no los realizan correctamente. A demás se les dificulta establecer y resolver problemas que involucren la letra como incógnita. En cuanto a las magnitudes directamente relacionadas lo estudiantes logran identificarlas, pero no las inversamente relacionadas. A partir de los resultados obtenidos se planteo la secuencia la cual lleva a la identificación de los métodos de solución de sistemas de ecuaciones lineales.

La primera situación planeada tenía como objetivos lograr que el estudiante identifique las ecuaciones lineales, la función lineal y la noción de pendiente. Lo cual se trabajó para reforzar el reconocimiento de las magnitudes directa e inversamente proporcionales y el despeje en. Esta situación se trabajo en las fases de acción, formulación y validación. Para luego los docentes hicieran la institucionalización de los objetos matemáticos e involucrados. En esta situación se trato de que la comprensión de la función lineal se diera por medio de los cambios de registro a partir de que los estudiantes vieran características de la tabla de datos, la grafica cartesiana y su expresión algebraica.

La segunda situación fundamental tenía como objetivos forjar en los estudiantes la capacidad de comprender en qué momentos es necesario utilizar un sistema de ecuaciones lineales, potenciar diversas maneras de solucionar un sistema de ecuaciones lineales y potenciar en los estudiantes la comprensión de los métodos gráfico, sustitución, igualación y reducción para solucionar sistemas de ecuaciones lineales. Igualmente que en la anterior fase los estudiantes trabajaron en las fases de acción, formulación y validación. Para que luego los docentes hicieran la institucionalización de los objetos matemáticos e involucrados. Finalmente se realizo una evaluación la cual permitió observar si los estudiantes adquirieron los conocimientos esperados.

\section{Logros y dificultades}

\section{Primera situación fundamental:}

- Los estudiantes hacen uso del lenguaje común para dar solución a la situación.

- Los estudiantes logran hacer cambios de registro, algunos con algunas dificultades dado la complejidad de estos.

- Se logró que los estudiantes reforzaran la manera de despejar una ecuación en 
- Se vio dificultad ya que los estudiantes querían llegar a una respuesta rápida o que el docente se la dirá, ya que se están acostumbrados a ejercicios de respuesta inmediata.

- Segunda situación fundamental:

- Se observo que los estudiantes intentaron establecer una sola ecuación lineal, cuando la situación establecía dos.

- Se llega en la fase de formulación a establecer las dos ecuaciones de enunciado problema, pero no se logra encontrara la estrategia que permita encontrar los valores de las incógnitas.

- Algunos estudiantes hacen uso de la investigación para dar solución al problema, llegando a identificar el método de igualación y sustitución.

En general se evidencio gran dificultad para llevar la situación fundamental, ya que los estudiante esperaban una clase magistral, y no ha construir su propio conocimientos, además de que no se esperaba que el docente los guiará por medio de preguntas y no dándole poco a poco la respuesta. Pero se logro que los estudiantes adquirieran un aprendizaje significativo de los métodos para resolver sistemas de ecuaciones lineales, además de que cada cual decidiera cual era el que iba a implementar.

\section{Reflexión final}

El ser un buen docente implica una reflexión contante al preparar clase, en la hora de la clase y después de dicha clase, es por eso que recomendamos que dicha reflexión no se haga solo al terminar la experiencia en el aula, sino a través de todo el proceso educativo, es decir en la fase preactiva, interactiva y post-activa. Es así como vemos la importancia de planear con anticipación el material destinado para la actividad con los estudiantes, planearse hipótesis de las producciones de los estudiantes y las posibles preguntas que como docentes podemos realizar para que los estudiantes logren construir su propio conocimiento. Ya que a partir de dicha planeación se podrán evitar inconvenientes en el aula de clases.

En cuanto a la interacción en el aula, es fundamental reconocer como docentes la importancia de que cada estudiante construya su propio conocimiento, ya que este va ser realmente significativo. En cuanto a la enseñanza de los métodos de resolución de un sistema de ecuaciones lineales es importante identificar la complejidad que estos implican, puesto que conllevan varios preconceptos que son necesarios que los estudiantes posean, para su aprendizaje y aplicación, así que es de vital importancia trabajar de la mano con los estudiantes, reconociendo las necesidades y la manera de aprendizaje de cada cual.

Finalmente se logra identificar a que es necesario hacer una ejercitación de procedimientos terminada esta secuencia, lo cual permitirá un mejor manejo del algoritmo de cada uno de estos métodos.

\section{Referencias}

Duval, R. (1999). Semiosis y pensamiento humano. Valle: Peter lang SA.Gallego, L., \& Fornés, L. (2009). Matemáticas: unidades didácticas. España: ESO.

Godino, J., \& Font, V. (2003). Razonamiento algebraico y su didáctica para maestros. Granada: Reprodigital.

Grupo Azarquiel. (1993). Ideas Y Actividades Para Enseñar álgebra. Madrid: Síntesis.

Haussler, P. (2003). Matemática para la administración y la economía. (Decima ed.). México: Pearson. 